\title{
Revisión por pares en la Revista Médica de Chile, año 2009
}

\author{
Peer-reviewers in Revista Médica de Chile, year 2009
}

\begin{abstract}
Los revisores externos de los manuscritos que se reciben en la Revista Médica de Chile cumplen una labor voluntaria, desinteresada y anónima (para los autores). La "revisión por pares" es uno de los pilares que sostienen la calidad de las revistas científicas y se ha hecho cada día más importante al aumentar la complejidad metodológica de los trabajos, en el área científica y en las especialidades clínicas. Esta colaboración demanda tiempo, esfuerzo, la revisión de bases de datos y de otras publicaciones, y la aplicación de un juicio valorativo, todo lo cual se sintetiza en un informe escrito que se pone a disposición de los editores. Es un trabajo que debe ser conocido y valorado por los lectores de la Revista y por las instituciones en que trabajan dichos revisores.
\end{abstract}

\begin{abstract}
Por ese motivo, una vez al año y en el número de julio de la Revista, se identifican las personas que durante el año precedente revisaron al menos un manuscrito. Durante 2009 contamos con la participación de más de 527 revisores externos, la mayoría de los cuales revisaron dos o tres manuscritos en el año. Quienes figuran en la nómina de Miembros del Comité Editorial Asesor de la Revista, revisaron generalmente un número aún mayor. Agradecemos su colaboración, en nombre de los editores de la Revista Médica de Chile y del Directorio de la Sociedad Médica de SantiagoSociedad Chilena de Medicina Interna.

La nómina que se publica a continuación es un documento que acredita su inapreciable participación en el proceso de "revisión por pares".
\end{abstract}

A

Abarca V, Katia
Abumohor G, Patricia
Acevedo B, Mónica
Acuña L, Guillermo
Acuña M, Raúl
Acuña P, Mónica
Acuña R, Julia
Aguayo G, Francisco
Aguayo N, Rubén
Aguilera O, Ledda
Alarcón M, Ana María
Albala B, Cecilia
Alfaro L, Jorge
Aller R, Felipe
Alliende R, María Angélica
Almagro L, Enrique
Álvarez G, Francisco
Álvarez A, Hernán
Álvarez A, María Isabel
Álvarez U, Gonzalo

Álvarez Z, Manuel

Alvo A, Miriam

Amat V, José

Amigo C, Hugo

Anabalón S, Patricio

Andrighetti C, Francisco

Anguita C, Timoleón

Apt B, Werner

Arancibia H, Francisco

Aranda C, Waldo

Araos H, Daniel

Aravena C, Teresa

Araya D, Fernando

Araya H, Iván

Araya Q, Magdalena

Araya Q, Verónica

Arellano H, Leonardo

Armas M, Rodolfo

Arteaga U, Eugenio

Arteaga H, Oscar

Arteaga Ll, Antonio

Asenjo G, René

\author{
Asenjo M, Sylvia \\ Assef C, Marcela \\ Astete A, Carmen Paz \\ Atalah S, Eduardo \\ Ayala R, Francisco \\ Aylwin H, Carmen Gloria
}

B

Badilla O, Lautaro

Baeza O, Aníbal

Bannura C, Guillermo

Baquerizo M, Antonio

Barros D, María Teresa

Barros M, Manuel

Bastías S, Gabriel

Beca I, Juan Pablo

Bedregal G, Paula

Behar A, Rosita

Behrens P, María Isabel

Bello S, Sergio

Bertín C, Pablo 
Besio R, Mauricio

Biagini A, Leandro

Bianchi S, Víctor

Binfa E, Lorena

Bitran C, Marcela

Blamey D, Rodrigo

Blanco C, Rafael

Blanco R, María Antonieta

Blümel M, Juan Enrique

Borja R, Hernán

Borzone T, Gisela

Brantes G, Sergio

Braun J, Sandra

Bravo L, Mireya

Bronfman F, Lucía

Browne L, Francisca

Bruhn C, Alejandro

Brunser T, Oscar

Bueno E, Beatriz

Bugedo T, Guillermo

Bustamante Z, Marco

Bustos M, Patricia

C

Cabello A, Hernán

Cabrera M, Raúl

Cabrera C, María Elena

Cáceres C, Texia

Campero S, Mario

Campusano M, Claudia

Canales R, Paulina

Canals L, Mauricio

Canals L, Claudio

Cano Sch, Francisco

Caorsi S, Carlos

Caro L, Jorge

Carrasco M, Carmen

Carreño T, Laura

Carvallo V, Aurelio

Carvajal C, Sergio

Carvajal H, Carlos

Cassorla G, Fernando

Castellón V, Enrique

Castillo M, Manuel

Castillo L, Cecilia

Castillo T, Silvia

Castro G, Pablo

Castro O, José

Catán G, Felipe

Caviedes S, Iván
Cerda A, Berta

Chamorro B, Hernán

Chaná C, Pedro

Chávez C, Pablo

Chávez G, Hernán

Chernilo S, Sara

Chomalí G, May

Cifuentes O, Lucía

Clavero R, José Miguel

Codoceo R, Verner

Comparini F, Beatriz

Contreras R, Adela

Contreras A, Marcela

Contreras M, Luis

Contreras P, Jaime

Contreras P, Juan Eduardo

Contreras S, Andrea

Cordella M, María Patricia

Cordero V, Miguel Angel

Cornejo R, Rodrigo

Corrales V, Raúl

Cortés G, Pablo

Cortés M, Fanny

Corvalán A, Camila

Cosoi Pérez, Eduardo

Crosby Russo, Javier

Crovari E, Fernando

Csendes J, Attila

Cuchacovich T, Miguel

Cuevas M, Ada

Cunill C, Eduardo

D

De Aretxabala U, Xabier

De la Fuente H, María

De la Maza C, María Pía

De la Sotta T, Raúl

Del Castillo S, César

Del Sol C, Mariano

Devoto C, Luigi

Díaz G, Rodrigo

Díaz A, Patricia

Díaz C, Carlos

Díaz C, Jaime

Díaz J, Juan Carlos

Díaz T, Violeta

Domenech L, Raúl

Dörr A, Anneliese

Dörr Z, Otto

D’Ottone M, Karin
Downey C, Patricio

Duclos H, Jaime

Duffau T, Gastón

Durruty A, Pilar

Dvoredsky B, Ana

$\underline{\mathbf{E}}$

Enríquez L, Octavio

Erazo R, Rodrigo

Escobar Ch, Carola

Espinoza G, Ricardo

Etérovic M, Ivo

F

Fajuri N, Alejandro

Fernández V, Alejandra

Ferreiro P, Osvaldo

Fielbaum C, Oscar

Figueroa C, Gustavo

Figueroa $\mathrm{G}$, Guillermo

Figueroa M, Claudia

Florenzano U, Fernando

Florenzano U, Ramón

Fluxá G, Fernando

Franco S, Carmen

Fritsch M, Rose Marie

Fuentealba P, Carlos

Fuentes G, Patricio

Fuentes M, Ximena

Fullerton U, Claudio

G

Gac E, Homero

Gai H, María Nella

Gajardo G, Gonzalo

Gajardo L, Héctor

Gajardo N, Jorge

Gálvez G, Sergio

Gambra A, Pilar

García B, Hernán

García-Huidobro T, Juan Pablo

Gatica R, Héctor

Gejman E, Roger

Gil D, Rodrigo

Giordano B, José

Gloger K, Sergio

Godoy F, Jaime

Godoy J, Gonzalo

Godoy O, Jorge 
Goecke S, Anneliese

Goic G, Alejandro

Gómez L, Fernando

Gómez M, Patricia

González B, Sergio

González C, Carlos

González H, Jorge

González G, María Isabel

González T, Matías

González V, Gilberto

Gotteland, Martín

Gramegna S, Gloria

Grant D, Carlos

Greig U, Douglas

Grez A, Mónica

Guajardo L, Jorge

Guarda S, Eduardo

Guerrero L, Marco

Guiraldes C, Ernesto

Guivernau B, Mauricio

Gutiérrez C, Jorge

Gutiérrez T, Miguel

Guzmán B, Leonardo

Guzmán D, Ana María

\section{H}

Halpern G, Muriel

Hanne A, Christel

Hepp D, Juan

Hermansen T, Carlos

Hermosilla D, Germán

Hernández F, Denisse

Hernández G, Guillermo

Hernández V, Rodrigo

Herskovic M, Viviana

Herskovic L, Pedro

Hidalgo V, Soledad

Hinzpeter K, Daniel

Hodgson B, María Isabel

Holzer M, Freddy

Honeyman M, Juan

Hoppe W, Arnold

Horwitz C, Nina

Hoyl M, Trinidad

Huidobro A, Christian

\section{I}

Ibáñez A, Luis

Ibáñez G, Pilar

Ibarra V, Álvaro
Icaza N, Gloria

Irribarra P, Verónica

Isamitt $\mathrm{D}$, Dionis

Iturriaga R, Hernán

Ivanovic-Zuvic R, Fernando

J

Jadresic M, Enrique

Jadue $\mathrm{H}$, Liliana

Jalil M, Jorge

Jara G, Alejandra

Jaramillo M, Arturo

Jarufe C, Nicolás

Jelves $\mathrm{M}$, Ivonne

Jensen B, Cristián

Jirón V, María Isabel

Jofré P, Pamela

Juri C, Carlos

\section{K}

Kahn Ch, Mariana

Kakarieka W, Elena

Kamann C, Francisco

Klaassen L, Julieta

Koppmann A, Alejandro

Kottow L, Miguel

\section{L}

Labarca L, Jaime

Labra J, Juan

Lahsen M de la P, Rodolfo

Lama T, Alexis

Larraguibel Q, Marcela

Lastra T, Jorge

Latorre M, Ricardo

Lecannelier A, Felipe

Lema F, Guillermo

León B, Lorena

Leisewitz V, Thomas

Letelier S, Luz María

Liberman G, Claudio

Lioi C, Ximena

Llanos L, Osvaldo

Llorens S, Pedro

López K, Francisco

López M, José Manuel

López N, Mercedes

López S, Gloria
López V, Néstor

Lubascher C, Jaime

Luchsinger F, Vivian

M

Macho F, Pilar

Madrid A, Jorge

Maggiolo G, Pedro

Maiz G, Alberto

Majlis L, Alejandro

Maluenda G, Fernando

Mansilla P, María Susana

Marchetti P, Nella

Mardones S, Francisco

Margozzini M, Paula

Marinovic M, Angélica

Martínez F, Claudio

Martínez S, Alejandro

Martínez T, María Angélica

Massardo V, Loreto

Matus C, Patricia

Mazzei P, Marinella

Medina C, Eduardo

Mellado T, Patricio

Melo G, Iván

Mena G, Francisco

Méricq G, Verónica

Mertens M, Renato

Meruane S, Jorge

Mesa L, Tomás

Mezzano A, Sergio

Michaud Ch, Patricio

Minguell U, José

Miquel P, Juan Francisco

Miranda C, Marcelo

Miranda C, Pedro

Mocarquer M, Alfredo

Moenne B, Karla

Molina C, Ramiro

Montenegro A, Hernán

Montero C, Edson

Montero L, Joaquín

Moore, Philippa

Morales M, Helia

Morales E, Sergio

Morales D, Marta

Moreno B, Rodrigo

Moreno G, Gladys

Moreno G, Manuel

Morris C, Raimundo 
Moyano D, Emilio

Moyano Sch, Leonor

Munizaga C, Fernando

Muñoz C del V, Patricia

Muñoz N, Sergio

Muñoz R, Mario

Muzzo B, Santiago

\section{$\mathrm{N}$}

Nazer H, Julio

Nazzal N, Carolina

Neira Q, Oscar

Norero V, Colomba

Noriega R, Luis Miguel

Novoa S, Fernando

\section{O}

Ocqueteau T, Mauricio

Olavarría L, Verónica

Olivos O, Cristina

Olmos C, Alfonso

Orlandi J, Luis

Ortiz P, Armando

Oviedo G, Sofía

Oyonarte G, Miguel

\section{$\mathbf{P}$}

Pacheco R, Daniel

Padilla E, Carlos

Palacios A, Andrés

Palacios M, Silvia

Palavecino Silva, Ricardo

Parra G, Juan Andrés

Passalacqua R, Walter

Peirano C, Patricio

Pereira G, Jaime

Pérez A, Claudia

Pérez B, Francisco

Pérez C, Carlos

Pérez H, María Angélica

Pérez P, Gilberto

Pertuzé R, Julio

Pezzani V, Marcela

Pimentel M, Fernando

Pineda A, Fernando

Pinedo M, George

Pino Z, Paulina

Pinto C, Juan

Pizarro S, Héctor
Poggi M, Helena

Pollak C, Felipe

Poniachik T, Jaime

Prieto D, Juan Carlos

Puelma P, Alvaro

Puga P, Julio

Püschel I, Klaus

\section{Q}

Quera P, Rodrigo

Quezada L, Arnoldo

Quijada H, Mario

Quiñones S, Luis

Quiroga G, Teresa

\section{$\mathbf{R}$}

Radrigán A, Francisco José

Raggio M, Ximena

Ramírez M, Verónica

Repetto L, Paula

Retamal C, Pedro

Reyes S, Eliana

Ribalta L, Gloria

Riedemann G, Pablo

Rioseco ST, Pedro

Rivero D, Santiago

Roa E, Iván

Roa S, Juan Carlos

Rodrigo S, Ramón

Rodríguez M, Jimena

Rodríguez D, Juan Carlos

Rodríguez G, Cristián

Rodríguez P, José Adolfo

Rodríguez T, Jorge

Roessler B, Emilio

Rojas G, Álvaro

Rojas P, Hilda

Rojas S, Nina

Román R, Rossana

Romero S, María Inés

Rossel M, Víctor

Rosselot J, Eduardo

Ruiz A, Inés

Ruz O, Manuel

S

Sabbagh P, Eduardo

Sáenz F, Roque

Salamanca F, Lucía
Salas P, Sofía

Saldías P, Fernando

Salvo C, Karen

Sánchez H, Alfonso

Sandoval R, Patricio

Sanhueza C, Patricio

Sanhueza R, Emilia

Santín M, Julia

Santos A, Manuel

Sanz C, Patricia

Sapunar Z, Jorge

Schiattino L, Irene

Schultz H, Marcela

Segovia D, Isabel

Segovia M, Roberto

Segovia R, Erico

Seitz C, Juan

Selman A, Rafael

Selman A, Alberto

Sepúlveda J, Rafael

Sepúlveda M, Ricardo

Sepúlveda N, Andrea

Sepúlveda R, Juan Enrique

Serani M, Alejandro

Serrano L, Valentina

Silva F, Claudio

Silva I, Hernán

Silva M, Sandra

Silva S, Juan Jorge

Silva V, Víctor

Silva Z, Claudio

Sirhan N, Marisol

Snow, Vincenza T.

Solís O, Iván

Soto F, Rodrigo

Soto I, Néstor

Soto S, Juan Ramón

Soto S, Francisco Javier

Sotomayor S, María Angélica

Soza M, Marco Antonio

Spotorno O, Angel

Squella B, Freddy

Stuardo L, Andrés

Suazo G, Iván

Sulbrandt C, José

Szántho P, György

T

Talesnik G, Eduardo

Tapia I, Jorge 
Tenhamm F, Eugenio

Thompson M, Luis

Toche P, Paola

Tomicic F, Vinko

Triviño B, Ximena

Trujillo V, Sergio

Turner G, Eduardo

U

Ulloa F, María Teresa

Unanue M, Nancy

Undurraga $\mathrm{P}$, Alvaro

Undurraga S, María Soledad

Uriarte $\mathrm{G}$ de C, Polentzi

Uribe M, Mario

Urrutia F, Lilian

Urzúa U, Jorge

V

Vacarezza Y, Ricardo

Valbuena M, José Rafael

Valdés L, Verónica
Valdés S, Gloria

Valdivia C, Gonzalo

Valdivieso D, Vicente

Valenzuela B, María Teresa

Valenzuela E, Jorge

Valenzuela M, Raúl

Valenzuela P, Sergio

Valenzuela Y, Carlos

Vargas M, Sergio

Vargas C, Nelson

Vásquez T, Patricia

Velasco P, Alfredo

Véliz L, Jesús

Verdugo L, Renato

Vera C, Aldo

Vergara S, Ismael

Vergara E, Fernando

Vergara I, Marcos

Vicente P, Benjamín

Villafranca A, Carlos

Villarroel D, Luis

Villaseca H, Miguel

Vogdt, Joachim

Vukasovic R, José Luis
W

Wainstein G, Eduardo

Weil P, Kristina

Wolff R, Marcelo

Y

Yáñez L, Alfredo

Yovanovich S, Jorge

Z

Zagolín B, Mónica

Zalaquett S, Ricardo

Zapata L, Rodrigo

Zárate B, Víctor

Zehnder B, Carlos

Zilleruelo B, Raúl

Zorrilla F, Sergio

Zúñiga G, Manuel

Zúñiga D, Alvaro 